\title{
$w$-b-Cone Distance and Its Related Results: A Survey
}

\author{
Reza Babaei ${ }^{1}$, Hamidreza Rahimi ${ }^{1, *}$, Manuel De la Sen ${ }^{2, *}$ (D) and and Ghasem Soleimani $\operatorname{Rad}^{1}$
}

1 Department of Mathematics, Faculty of Science, Central Tehran Branch, Islamic Azad University, Tehran 512, Iran; rez.babaei.sci@iauctb.ac.ir (R.B.); gha.soleimani.sci@iauctb.ac.ir (G.S.R.)

2 Institute of Research and Development of Processes IIDP, University of the Basque Country, Campus of Leioa, P.O. Box 48940 Leioa (Bizkaia), Spain

* Correspondence: rahimi@iauctb.ac.ir (H.R.); manuel.delasen@ehu.eus (M.D.l.S.)

Received: 6 December 2019; Accepted: 13 January 2020; Published: 16 January 2020

\begin{abstract}
In this work, we define the concept of a $w$-b-cone distance in $t v s$-cone $b$-metric spaces which differs from generalized $c$-distance in cone $b$-metric spaces, and we discuss its properties. Our results are significant, since all of the results in fixed point theory with respect to a generalized $c$-distance can be introduced in the version of $w$-b-cone distance. Moreover, using Minkowski functionals in topological vector spaces, we prove the equivalence between some fixed point results with respect to a $w$ t-distance in general $b$-metric spaces and a $w$-b-cone distance in $t v s$-cone $b$-metric spaces.
\end{abstract}

Keywords: $w$-b-cone distance; tvs-cone $b$-metric space; lower $b$-semicontinuous; Minkowski functional

MSC: AMS subject classification 2000: primary 47H10, 45B05; secondary 54H25

\section{Introduction and Preliminaries}

In 1931, Wilson [1] introduced symmetric spaces, as metric-like spaces lacking the triangle inequality. Thereinafter, $b$-metric spaces or metric type spaces as a new kind of spaces were defined by Bakhtin [2] and Boriceanu [3].

Definition $1([2,3])$. Let $X$ be a nonempty set. A real-valued function $D: X \times X \rightarrow[0, \infty)$ is said to be a $b$-metric if the following properties hold:

$\left(D_{1}\right) \quad D(x, y)=0$ iff $x=y$ for all $x, y \in X$;

$\left(D_{2}\right) \quad D(x, y)=D(y, x)$ for all $x, y \in X$;

$\left(D_{3}\right) \quad D(x, z) \leq s[D(x, y)+D(y, z)]$ for all $x, y, z \in X$.

With this definition, $(X, D)$ is named a b-metric space. Obviously, a b-metric space with $s=1$ is a metric space.

Then, they introduced some definitions and topological properties in $b$-metric spaces and obtained some applications of these spaces in nonlinear analysis (also, see [4,5]). In 1996, Kada et al. [6] considered a $w$-distance in metric spaces for solving some non-convex minimization problems. Then some authors $[7,8]$ applied this concept in fixed point theory.

Definition 2 ([6]). Consider a metric space $(X, d)$. Let $\rho: X \times X \rightarrow[0,+\infty)$ be a function satisfying the following conditions:

$\left(\rho_{1}\right) \quad \rho(x, z) \leq \rho(x, y)+\rho(y, z)$ for all $x, y, z \in X$;

$\left(\rho_{2}\right) \quad \rho$ is lower semicontinuous in its second variable; that is, if $x \in X$ and $y_{n} \rightarrow y$ in $X$ then $\rho(x, y) \leq$ $\liminf _{n} \rho\left(x, y_{n}\right)$;

$\left(\rho_{3}\right) \quad$ for each $\varepsilon>0$, there exists $\delta>0$ such that $\rho(z, x)<\delta$ and $\rho(z, y)<\delta$ imply that $d(x, y)<\varepsilon$. 
Then $\rho$ is named a w-distance on $X$.

In 2014, Hussain et al. [9] generalized this $w$-distance to $w t$-distance on a $b$-metric space and obtained several its properties.

Definition 3 ([9]). Consider a b-metric space $(X, D)$ with $s \geq 1$. Let $p: X \times X \rightarrow[0,+\infty)$ be a function satisfying the following conditions:

$\left(w_{1}\right) \quad p(x, z) \leq s[p(x, y)+p(y, z)]$ for all $x, y, z \in X$;

$\left(w_{2}\right) \quad p$ is lower b-semicontinuous in its second variable; that is, if $x \in X$ and $y_{n} \rightarrow y$ in $X$ then $p(x, y) \leq$ $s \liminf \operatorname{in}_{n} p\left(x, y_{n}\right)$;

$\left(w_{3}\right)$ for each $\varepsilon>0$, there exists $\delta>0$ such that $p(z, x)<\delta$ and $p(z, y)<\delta$ imply that $D(x, y)<\varepsilon$.

Then $p$ is named a wt-distance on $X$.

Obviously, a $w t$-distance in a $b$-metric space with $s=1$ is a $w$-distance in a metric space in the sense of Definition 2.

Ordered normed spaces and cones have many applications in applied mathematics. Hence, fixed point theory in K-normed and K-metric spaces was extended in the 20th century (see, e.g., [10-12]). In 2007, Huang and Zhang [13] reintroduced these spaces under the name of cone metric spaces by substituting the set of real number by an ordered normed space. Also, topological vector space-valued version of this concept was discussed in [14-16]. In 2011, analogously with the definition of $b$-metric spaces and cone metric spaces, Hussain and Shah [17] and Ćvetković et al. [18] defined cone $b$-metric spaces (or cone metric type spaces) and proved some topological and analysis properties (also, see [19]). Moreover, topological vector space-valued version of cone $b$-metric spaces was defined in [20]. On the other hands, in 2011, Cho et al. [21] and Wang and Guo [22] introduced a cone version of the $w$-distance which is named $c$-distance. Then, some fixed point results under $w$-distance in metric spaces and under $c$-distance in cone metric spaces and tvs-cone metric spaces were obtained in $[23,24]$ and the references cited therein. In 2015, Bao et al. [25] introduced a generalized c-distance in cone $b$-metric spaces and proved several fixed point theorems with respect to this generalized distance (also, see $[26,27])$.

We start with some preliminary definitions and results in $[10,28,29]$.

Let $E$ be a real Hausdorff topological vector space (for short, is named tvs) with the zero vector $\theta$. A proper closed subset $P \neq \varnothing$ of $E$ is said to be a cone if $P+P \subset P, \lambda P \subset P$ for $\lambda \geq 0$ and $P \cap(-P)=\{\theta\}$. For an arbitrary cone $P \subset E$, we consider a partial ordering $\preceq$ with respect to $P$ by $x \preceq y \Longleftrightarrow y-x \in P$. We say $x \prec y$ if $x \preceq y$ and $x \neq y$, and $x \ll y$ if and only if $y-x \in$ int $P$, where int $P$ is the interior of $P$. Also, the cone $P$ is called solid if int $P \neq \varnothing$. Now, the pair $(E, P)$ is an ordered tus. For a pair of elements $x, y \in E$ with $x \preceq y$, set $[x, y]=\{z \in E: x \preceq z \preceq y\}$. A subset $A$ of $E$ is called order-convex if $[x, y] \subset A$. By this definition, an ordered tos $(E, P)$ is order-convex if it has a base of neighborhoods of $\theta$ consisting of order-convex subsets. In this case, $P$ is named a normal cone. If $E$ is a normed space, this condition means that the unit ball is order-convex; that is, there is a number $K$ such that for all $x, y \in E, \theta \preceq x \preceq y$ imply $\|x\| \leq K\|y\|$.

Theorem 1 ([11]). Let the underlying cone of an ordered tvs be normal and solid. Then tvs is an ordered normed space.

Below, we provide a full explanation of the main motivation behind this project: In 2010, Du [14] studied the equivalence of vectorial versions of fixed point theorems in cone metric spaces and scalar versions of fixed point theorems in general metric spaces. Especially, he proved the equivalence between Banach contraction principle in general metric spaces and in tvs-cone metric spaces by a nonlinear scalarization function. Almost simultaneously with Du's work, in 2011, Kadelburg et al. [30] showed that the same results can be obtained more easily using a Minkowski 
functional in topological vector spaces. In the meantime, Ćirić et al. [31] defined a $w$-cone distance in tvs-cone metric spaces, which is a generalization of the $w$-distance introduced by Kada et al. [6]. This definition is differ of the definition of the $c$-distance introduced by Cho et al. [21] and Wang and Guo [22]. Indeed, each $w$-cone distance is a $c$-distance, but the converse is not true. Also, Ćirić et al. noted that the method of Du for cone contraction mappings cannot be applied for a $w$-cone distance contraction mappings. Then they proved some fixed point theorems with respect to a $w$-cone distance in tvs-cone metric spaces. Also, at the end of their paper, they posed an open question about the existence and uniqueness of fixed point of quasi-contraction mappings by applying a $w$-cone distance. In the same year, Kadelburg and Radenović [32] answered this question by using a similar technique in [30]. On the other hands, in 2013, Du and Karapinar [20] studied about the equivalence of vectorial versions of fixed point theorems in cone $b$-metric spaces and scalar versions of fixed point theorems in $b$-metric spaces. Now, let us we define a tvs-cone $b$-metric space, which is introduced by Kadelburg et al. [19] and Du and Karapinar [20].

Definition 4 ([19]). Let $X$ be a nonempty set, $s \geq 1$ and $(E, P)$ be an ordered tos. A function $d: X \times X \rightarrow P$ is said to be a tos-cone $b$-metric if the following properties hold:

$\left(d_{1}\right) \quad d(x, y)=\theta$ iff $x=y$ for all $x, y \in X ;$

$\left(d_{2}\right) \quad d(x, y)=d(y, x)$ for all $x, y \in X$;

$\left(d_{3}\right) \quad d(x, z) \preceq s[d(x, y)+d(y, z)]$ for all $x, y, z \in X$.

With this definition, $(X, d)$ is named a tos-cone $b$-metric space.

Now, by applying Definition 4, we can obtain other version of metric spaces as following.

Remark 1. Obviously, a tos-cone b-metric space with $s=1$ is a tvs-cone metric space in the sense of [14]. If we replace $E$ by a real Banach space in Definition 4, we get the cone b-metric space in the sense of [17,18]. Now, if we consider $s=1$ then we can obtain the classic definition of cone metric space introduced by Huang and Zhang [13] (also, see [33]). Moreover, it is evident that our definition coincides with the definition of b-metric spaces (Definition 1) if we replace $E$ by the set of real numbers and $P$ by the set of nonnegative real numbers. In this case, if we consider $s=1$ then we obtain the same well-known results of metric spaces. Therefore, it seems Definition 4 is the most complete version of type metric spaces.

Definition $5([20,23,34])$. Let $\left\{x_{n}\right\}$ be a sequence in a tos-cone $b$-metric space $(X, d)$. Then

- $\left\{x_{n}\right\}$ converges to $x$ if for every $c \in E$ with $\theta \ll c$ there exist $n_{0} \in \mathbb{N}$ such that $d\left(x_{n}, x\right) \ll c$ for all $n>n_{0}$, and we write $x_{n} \rightarrow x$ as $n \rightarrow \infty$.

- $\left\{x_{n}\right\}$ is called a Cauchy sequence if for every $c \in E$ with $\theta \ll c$ there exist $n_{0} \in \mathbb{N}$ such that $d\left(x_{n}, x_{m}\right) \ll c$ for all $m, n>n_{0}$.

- $\quad \mathrm{X}$ is said to be complete if every Cauchy sequence in $\mathrm{X}$ is convergent in $\mathrm{X}$.

Also, we apply the following property several times in the proof of our theorems:

(*) Let $v \preceq \lambda v$ with $v \in P$ and $0 \leq \lambda<1$. Then $v=\theta$.

In [19], Kadelburg et al. showed that the same results of Du and Karapinar [20] can be obtained more easily using a Minkowski functional in tvs-cone $b$-metric spaces.

In this work, we first define a $w$-b-cone distance in the framework of $t v s$-cone $b$-metric spaces. Then we introduce several its preliminary properties. As an application, we obtain several fixed point and periodic point theorems for contractive mappings in such spaces with a non-normal cone under contractive conditions introduced in the terms of $w-b$-cone distance. Furthermore, we prove the equivalence of vectorial versions of fixed point theorems with respect to a $w-b$ cone distance in cone $b$-metric spaces and scalar versions of fixed point theorems with respect to a $w t$-distance in $b$-metric 
spaces. In fact, this research is a continuation of the former articles such as $[6,14,20-22,30-32]$ which extended, generalized and unified all of them in this literature.

\section{Main Results}

Let's start by introducing the following definition.

Definition 6. Let $(X, d)$ be a tos-cone b-metric space. A real-valued function $f: X \rightarrow P$ is said to be lower $b$-semicontinuous at a point $x \in X$ if for each $c \in$ int $P$, there is $n_{0} \in \mathbb{N}$ such that $f(x) \preceq s f\left(x_{n}\right)+c$ for all $n \geq n_{0}$, where $\left\{x_{n}\right\}$ is a sequence in $X$ and $x_{n} \rightarrow x$.

Now, we introduce the following new definition of distance version in tos-cone $b$-metric spaces. The idea of this definition comes from the definition of $w$-cone distance was introduced by Ćirić et al. [31] and the definition of generalized $c$-distance in cone $b$-metric spaces was defined by Bao et al. [25] and Soleimani Rad et al. [34].

Definition 7. Consider a tos-cone b-metric space $(X, d)$ with $s \geq 1$. Let $q: X \times X \rightarrow P$ be a function satisfying the following properties:

$\left(q_{1}\right) \quad q(x, z) \preceq s[q(x, y)+q(y, z)]$ for all $x, y, z \in X$;

$\left(q_{2}\right) \quad q(x, \cdot): X \rightarrow P$ is lower $b$-semicontinuous for all $x \in X$;

$\left(q_{3}\right)$ for all $\theta \ll c$, there exists $\theta \ll e$ such that $q(z, x) \ll e$ and $q(z, y) \ll e$ implying $d(x, y) \ll c$.

Then $q$ is said to be a $w$-b-cone distance on $X$.

Remark 2. Applying the definition of c-sequence, the condition $\left(q_{2}\right)$ of above definition can be considered equivalently as follow:

$\left(q_{2}^{\prime}\right) \quad$ If $y_{n}, y \in X, y_{n} \rightarrow y$ as $n \rightarrow \infty$ and $g(y)=q(x, y)$, then $g(y)-g\left(y_{n}\right)$ is a c-sequence.

Remark 3. Each wt-distance in a b-metric space (in the sense of Hussain et al. [9]) is w-b-cone distance in the tos-cone $b$-metric space. Also, for $s=1, a w$-b-cone distance is a w-cone distance of [31]. In this manner, let $E=\mathbb{R}$ and $P=[0, \infty)$. Then we have the same definition of w-distance of Kada et al. [6].

Remark 4. Note that the definition of generalized c-distance of Bao et al. [25] and the definition of generalized $c$-distance of Soleimani Rad et al. [34] differ from Definition 7 of $w$-b-cone distance. Also, it is evident that every $w$-b-cone distance is a $c$-distance, but the reverse is not true.

Now, we give some examples in the framework of tvs-cone $b$-metric spaces.

Lemma 1. If $(X, d)$ be a cone $b$-metric space, then the cone $b$-metric $d$ is a $w$-b-cone distance $q$.

Proof. Note that if $q=d$, then $q$ satisfies $\left(q_{1}\right)$. Thus we only prove $\left(q_{2}\right)$ and $\left(q_{3}\right)$. Let $x, y, y_{n} \in X$, $y_{n} \rightarrow y$, and $c \in E$ with $c \in$ int $P$ be arbitrary. Since $y_{n} \rightarrow y$, there exists $N \in \mathbb{N}$ such that $d\left(y_{n}, y\right) \preceq \frac{c}{s}$ for each $n \geq N$. Now, if $G(y)=d(x, y)$, then

$$
G(y)=d(x, y) \preceq s\left[d\left(x, y_{n}\right)+d\left(y_{n}, y\right)\right] \preceq s d\left(x, y_{n}\right)+c=s G\left(y_{n}\right)+c
$$

for all $n \geq n_{0}$. Hence, $q(x, \cdot)=d(x, \cdot)$ is lower $b$-semicontinuous and $\left(q_{2}\right)$ is established. For proving $\left(q_{3}\right)$, for each $c \in \operatorname{int} P$, set $e=\frac{c}{2 s}$. Then $q(z, x) \ll e$ and $q(z, y) \ll e$ imply that $d(x, y) \ll c$. This completes the proof.

Example 1. Let $(X, d)$ be a tos-cone $b$-metric space and $u \in X$ fixed. Then $q(x, y)=\frac{1}{s} d(u, y)$ is a $w$-b-cone distance. Indeed, we have $s q(x, z)=d(u, z) \preceq s^{2}(d(u, y)+d(u, z))$, i.e., $q(x, z) \preceq s q(x, y)+s q(y, z)$. Thus, 
$\left(q_{1}\right)$ is true. Similar to Lemma 2, one can prove the validity of condition $\left(q_{2}\right)$. Finally, $\left(q_{3}\right)$ is obtained by taking $e=\frac{c}{2 s^{2}}$.

Example 2. Let $E=C_{\mathbb{R}}[0,1]$, max-norm, $P=\{f \in E: f(t) \geq 0$ for $t \in[0,1]\}$, $\tau^{*}$ and $X$ are as in same Example 3 of [23]. Also, define the cone b-metric $d: X \times X \rightarrow\left(E, \tau^{*}\right)$ by $d(x, y)(t)=|x-y|{ }^{s} \varphi(t)$ with $s \in\{1,2\}$, where $\varphi \in P$ is a fixed element. Then

$$
q_{1}(x, y)(t)=d(x, y)(t), \quad q_{2}(x, y)(t)=y^{s} \varphi(t) \quad \text { and } \quad q_{3}(x, y)(t)=\left(x^{s}+y^{s}\right) \varphi(t)
$$

are three examples of $w$-b-cone distances in a tos-cone b-metric space.

From above examples, for all $x, y \in X$, we conclude that

- $q(x, y)=q(y, x)$ is not presently true;

- $q(x, y)=\theta$ does not certainly equivalent to $x=y$.

Lemma 2. Consider a tvs-cone $b$-metric space $(X, d)$ with $w$-b-cone distance $q$ on $X$. Also, let $\left\{\alpha_{n}\right\}$ be a sequence in $X$ and $\alpha, \beta, \gamma \in X$, and $\left\{x_{n}\right\}$ and $\left\{y_{n}\right\}$ be two c-sequences in $P$. Then

$\left(l_{1}\right) \quad$ If $q\left(\alpha_{n}, \beta\right) \preceq x_{n}$ and $q\left(\alpha_{n}, \gamma\right) \preceq y_{n}$ for $n \in \mathbb{N}$, then $\beta=\gamma$. Specially, if $q(\alpha, \beta)=\theta$ and $q(\alpha, \gamma)=\theta$, then $\beta=\gamma$.

$\left(l_{2}\right) \quad$ If $q\left(\alpha_{n}, \alpha_{m}\right) \preceq x_{n}$ for $m>n>n_{0}$ (for some $\left.n_{0} \in \mathbb{N}\right)$, then $\left\{\alpha_{n}\right\}$ is a Cauchy sequence.

Proof. Since the proof is easy and similar as in the case of $w$-distance in tvs-cone metric spaces in [31], we omit it.

Now, we prove some fixed and periodic point results with respect to a $w$-b-cone distance. Note that the scalarization method of Du and Karapinar [20] for linear contractive condition cannot be considered for a linear $w$-b-cone distance contractive condition. Thus, our results are substantial and new. In the following theorem, which extends and improves Theorem 9 of [31], Theorem 4.2 of [9] and Theorem 2 of [6], we obtain an estimate for a $w$-b-cone distance $q\left(x_{n}, z\right)$ of an approximate value $x_{n}$.

Theorem 2. Consider a complete tos-cone $b$-metric space $(X, d)$ with $w$-b-cone distance $q$ on $X$. Assume that $f: X \rightarrow X$ is a mapping such that

$$
q\left(f x, f^{2} x\right) \preceq k q(x, f x)
$$

for all $x \in X$, where $0 \leq k<\frac{1}{s}$. Suppose that one of the following assertions holds:

(i) If $f y \neq y$, there exists $c \in$ int $P$ with $c \neq \theta$ such that $c \ll q(x, y)+q(x, f x)$ for each $x \in X$;

(ii) $f$ is continuous.

Then $z \in X$ is a fixed point of $f$ and

$$
q\left(f^{n} x_{0}, z\right) \preceq \frac{s^{2} k^{n}}{1-s k} q\left(x_{0}, f x_{0}\right)
$$

for all $n \in \mathbb{N}$. Moreover, if $f u=u$ for some $u \in X$, then $q(u, u)=\theta$.

Proof. Let $x_{0} \in X$ and $x_{n}=f x_{n-1}=f^{n} x_{0}$. If $x_{n_{0}+1}=x_{n_{0}}$ for some $n_{0} \in \mathbb{N}$, then $x_{n_{0}}$ is a fixed point of $f$. Otherwise, we obtain from (1) that

$$
\begin{aligned}
q\left(x_{n}, x_{n+1}\right) & =q\left(f x_{n-1}, f^{2} x_{n-1}\right) \preceq k q\left(x_{n-1}, f x_{n-1}\right) \\
& =k q\left(f x_{n-2}, f^{2} x_{n-2}\right) \preceq k^{2} q\left(x_{n-2}, f x_{n-2}\right) \preceq \cdots \preceq k^{n} q\left(x_{0}, x_{1}\right) .
\end{aligned}
$$


Hence, for all $m, n \in \mathbb{N}$ with $m>n$, it follows from (3) and $\left(q_{1}\right)$ that

$$
\begin{aligned}
q\left(x_{n}, x_{m}\right) & \preceq s\left[q\left(x_{n}, x_{n+1}\right)+q\left(x_{n+1}, x_{m}\right)\right] \\
& s q\left(x_{n}, x_{n+1}\right)+s\left[s\left[q\left(x_{n+1}, x_{n+2}\right)+q\left(x_{n+2}, x_{m}\right)\right]\right] \\
& \vdots \\
& \preceq s q\left(x_{n}, x_{n+1}\right)+s^{2} q\left(x_{n+1}, x_{n+2}\right)+\cdots+s^{m-n} q\left(x_{m-1}, x_{m}\right) \\
& \preceq\left(s k^{n}+s^{2} k^{n+1} \cdots+s^{m-n} k^{m-1}\right) q\left(x_{0}, x_{1}\right) \\
& \preceq \frac{s k^{n}}{1-s k} q\left(x_{0}, x_{1}\right) .
\end{aligned}
$$

Thus, by applying Lemma $2\left(l_{2}\right),\left\{x_{n}\right\}$ is a Cauchy sequence in complete space $X$. Hence, there exists a point $z \in X$ such that $x_{n} \rightarrow z$ as $n \rightarrow \infty$.

Now, we will prove the estimate (2). Consider a function $g: X \rightarrow P$ by $g(x)=q\left(x_{n}, x\right)$ for each $n \in \mathbb{N}$. Consider $\left(q_{2}\right)$ and (1). Since $x_{n} \rightarrow z$ as $n \rightarrow \infty$, there is $M \in \mathbb{N}$ such that $q\left(x_{n}, z\right) \preceq$ $s q\left(x_{n}, x_{m}\right)+c$ for $m>M$ and for each $c \in \operatorname{int} P$. Now, it follows from (4) that

$$
q\left(f^{n} x_{0}, z\right)=q\left(x_{n}, z\right) \preceq \frac{s^{2} k^{n}}{1-s k} q\left(x_{0}, f x_{0}\right)+c .
$$

If we consider $c=\frac{c}{j}$ for some $j \in \mathbb{N}$, then we obtain

$$
\frac{s^{2} k^{n}}{1-k} q\left(x_{0}, f x_{0}\right)+\frac{c}{j}-q\left(f^{n} x_{0}, z\right) \in P .
$$

It is easy to show that

$$
\lim _{j \rightarrow \infty}\left(\frac{s^{2} k^{n}}{1-k} q\left(x_{0}, f x_{0}\right)+\frac{c}{j}-q\left(f^{n} x_{0}, z\right)\right)=\frac{s^{2} k^{n}}{1-k} q\left(x_{0}, f x_{0}\right)-q\left(f^{n} x_{0}, z\right) .
$$

Since $P$ is a closed set, we have

$$
\frac{s^{2} k^{n}}{1-k} q\left(x_{0}, f x_{0}\right)-q\left(f^{n} x_{0}, z\right) \in P,
$$

which implies that $q\left(f^{n} x_{0}, z\right) \preceq \frac{s^{2} k^{n}}{1-s k} q\left(x_{0}, f x_{0}\right)$. Thus, (2) is established.

At the first, suppose that $(i)$ is satisfied. Then we show that $f z=z$. Let $f z \neq z$ (to the contrary). Then, from (i), we have

$$
c \ll q(x, z)+q(x, f x)
$$

for all $x \in X$, where $\theta \ll c$. Now, using (2), (4) and (5), there exists $n_{0} \in \mathbb{N}$ such that

$$
q\left(x_{n}, z\right) \ll \frac{c}{4} \quad, \quad q\left(x_{n}, x_{n+1}\right) \ll \frac{c}{4}
$$

for all $n>n_{0}$. Now, let $x=x_{n}$. It follows from (6), (7), $\left(q_{2}\right)$ and the convergence $x_{n}$ to $z$ that

$$
c \ll q\left(x_{n}, z\right)+q\left(x_{n}, x_{n+1}\right) \ll \frac{c}{4}+\frac{c}{4}=\frac{c}{2},
$$

which is a contradiction (because $\theta \neq c \in \operatorname{int} P$ ). Thus, our guess about $f z \neq z$ is false. Hence, $f z=z$. 
Now, suppose that (ii) holds. By applying continuity of $f$, convergence $x_{n}$ to $z$ and the uniqueness of limit, we have $f z=z$. To complete the proof, let $f u=u$. It follows from (1) that

$$
q(u, u)=q\left(f u, f^{2} u\right) \preceq k q(u, f u)=k q(u, u) .
$$

Since $0 \leq k<\frac{1}{s}$ and $s \geq 1$, then (8) and $(*)$ imply that $q(u, u)=\theta$. This completes the proof.

Remark 5. Since all of other theorems in various version of distances can be obtained of Theorem 2, we claim our theorem is the most complete theorem with respect to a version of distance (see, Remarks 1 and 3). Also, using Theorem 2, we can obtain many fixed point results in tvs-cone b-metric spaces and especially in tvs-cone metric spaces by considering $s=1$. Consequently, we can prove all of them in metric fixed point theory without direct proof and also without using Du's method [14] (by considering $E=\mathbb{R}$ and $P=[0, \infty)$ ).

Question: Can the conditions (i) and (ii) be replaced by another condition in Theorem 2?

Corollary 1. Consider a complete tvs-cone $b$-metric space $(X, d)$ with $w$-b-cone distance $q$ on $X$. Assume that $f: X \rightarrow X$ is a mapping such that

$$
q\left(f x, f^{2} x\right) \preceq h q\left(x, f^{2} x\right)
$$

for all $x \in X$, where $0 \leq h<\frac{1}{s^{2}+s}$. If one of the assertions ( $i$ ) or (ii) in Theorem 2 holds, then $z \in X$ will be a fixed point of $f$. Further, if $f u=u$, then $q(u, u)=\theta$.

Proof. Let $x \in X$ is arbitrary. It follows from (9) and $\left(q_{1}\right)$ that

$$
q\left(f x, f^{2} x\right) \preceq h q\left(x, f^{2} x\right) \preceq h s\left[q(x, f x)+q\left(f x, f^{2} x\right)\right] .
$$

Thus, we have $(1-h s) q\left(f x, f^{2} x\right) \preceq h s q(x, f x)$, which implies that $q\left(f x, f^{2} x\right) \preceq k q(x, f x)$ with $0 \leq$ $k=\frac{h s}{1-h s}<\frac{1}{s}$. The proof further follows by Theorem 2 .

Corollary 2. Consider a complete tos-cone metric space $(X, d)$ with w-cone distance $q$ on $X$. Assume that $f: X \rightarrow X$ is a mapping such that $q\left(f x, f^{2} x\right) \preceq h q\left(x, f^{2} x\right)$ for all $x \in X$, where $0 \leq h<\frac{1}{2}$. If one of the assertions (i) or (ii) in Theorem 2 holds, then $z \in X$ will be a fixed point of $f$. Further, if $f u=u$, then $q(u, u)=\theta$.

Proof. In order to proof, set $s=1$ in Corollary 1 .

Clearly, if $z$ is a fixed point of $f$, then $z$ is a fixed point of $f^{n}$ as well for all $n \in \mathbb{N}$, but the reverse is not true. If a mapping $f: X \rightarrow X$ satisfies Fix $(f)=\operatorname{Fix}\left(f^{n}\right)$ for each $n \in \mathbb{N}$, where Fix $(f)$ is the set of fixed points of $f$, then $f$ has property $(P)$ [35-37]. The following theorem extends and improves Theorem 12 of Ćirić et al. [31], Theorem 4 of Đorđević [23] and Theorem 2 of Abbas and Rhoades [35].

Theorem 3. Consider a complete tvs-cone $b$-metric space $(X, d)$ with $w$-b-cone distance $q$ on $X$. Assume that the mapping $f: X \rightarrow X$ is subject to

$$
q\left(f x, f^{2} x\right) \preceq k q(x, f x)
$$

for all $x \in X$ with $0 \leq k<\frac{1}{s}$, then $f$ has property $(P)$. 
Proof. Obviously, if $f$ has a fixed point $z$, then $z$ is a fixed point of $f^{n}$ for each integer number $n$. Let $z \in$ Fix $\left(f^{n}\right)$; that is, $f^{n} z=z$. Then, from (10), we have

$$
\begin{aligned}
q(z, f z)=q\left(f^{n} z, f f^{n} z\right) & =q\left(f f^{n-1} z, f^{2} f^{n-1} z\right) \\
& \preceq k q\left(f^{n-1} z, f f^{n-1} z\right)=k q\left(f f^{n-2} z, f^{2} f^{n-2} z\right) \\
& \preceq k^{2} q\left(f^{n-2} z, f f^{n-2} z\right) \preceq \cdots \preceq k^{n} q(z, f z),
\end{aligned}
$$

which implies that $q(z, f z)=\theta$ (by $(*)$ and $0 \leq k<\frac{1}{s}$ ). Hence, for each $m \in\{1,2, \cdots, n\}$, we get $q\left(f^{m} z, f^{m+1} z\right) \preceq k^{m} q(z, f z)=\theta$. Thus, $q\left(f^{m} z, f^{m+1} z\right)=\theta$. Using $\left(q_{1}\right)$, we have

$$
q\left(z, f^{2} z\right) \preceq s\left[q(z, f z)+q\left(f z, f^{2} z\right)\right]=\theta,
$$

which implies that $q\left(z, f^{2} z\right)=\theta$. By following this process, we get $q\left(z, f^{n} z\right)=\theta$. Since $f^{n} z=z$, we have $q(z, z)=\theta$. Now, by applying Lemma 1. $\left(l_{1}\right)$, we conclude that $f z=z$; that is, $z$ is also a fixed point of $f$. Hence, $z \in \operatorname{Fix}(f)$ and so $\operatorname{Fix}\left(f^{n}\right) \subseteq \operatorname{Fix}(f)$. This ends to the proof.

Corollary 3. Consider a complete tos-cone metric space $(X, d)$ with w-cone distance $q$ on $X$. Assume that the mapping $f: X \rightarrow X$ is subject to

$$
q\left(f x, f^{2} x\right) \preceq k q(x, f x)
$$

for all $x \in X$ with $0 \leq k<1$, then $f$ has property $(P)$.

In the following theorem, we prove the existence of fixed point with respect to a $w$-b-cone distance for a contraction, which is included Banach contraction version, Kannan contraction version and Cho contraction version as you will see in the sequel.

Theorem 4. Consider a complete tvs-cone $b$-metric space $(X, d)$ with $w$-b-cone distance $q$ on $X$. Assume that $f: X \rightarrow X$ is a mapping such that

$$
q(f x, f y) \preceq \alpha_{1} q(x, y)+\alpha_{2} q(x, f x)+\alpha_{3} q(y, f y)+\alpha_{4} q(x, f y)
$$

for all $x, y \in X$, where $\alpha_{i} \geq 0$ for $i=1,2,3,4$ and

$$
s\left(\alpha_{1}+\alpha_{2}\right)+\alpha_{3}+\left(s^{2}+s\right) \alpha_{4}<1 .
$$

If either $(i) f$ is continuous, or (ii) there exists $c \in$ intP with $c \neq \theta$ such that

$$
c \ll q(x, y)+q(x, f x)+q(f x, y)
$$

for each $x, y \in X$ with $y \neq f y$, then $f$ has a fixed point. Further, if $f u=u$, then $q(u, u)=\theta$.

Proof. For arbitrary $x_{0} \in X$, consider the sequence $\left\{x_{n}\right\}$ with $x_{n}=f^{n} x_{0}$, where $n \in \mathbb{N}$. If $x_{n}=x_{n+1}$ for some $n$, then $x_{n}$ will be a fixed point of $f$. Hence, let $x_{n} \neq x_{n+1}$ for all $n \in \mathbb{N}$. Consider $x=x_{n-1}$ and $y=x_{n}$ in (11). By a simple calculation, we have

$$
q\left(x_{n}, x_{n+1}\right)=q\left(f x_{n-1}, f x_{n}\right) \preceq\left(\alpha_{1}+\alpha_{2}+s \alpha_{4}\right) q\left(x_{n-1}, x_{n}\right)+\left(\alpha_{3}+s \alpha_{4}\right) q\left(x_{n}, x_{n+1}\right),
$$

which implies that

$$
q\left(x_{n}, x_{n+1}\right) \preceq \frac{\alpha_{1}+\alpha_{2}+s \alpha_{4}}{1-\left(\alpha_{3}+s \alpha_{4}\right)} q\left(x_{n-1}, x_{n}\right)
$$


for all $n \in \mathbb{N}$. Now, by repeating the procedure, we obtain $q\left(x_{n}, x_{n+1}\right) \preceq \lambda^{n} q\left(x_{0}, x_{1}\right)$ for all $n \in \mathbb{N}$, where $0 \leq \lambda=\frac{\alpha_{1}+\alpha_{2}+s \alpha_{4}}{1-\left(\alpha_{3}+s \alpha_{4}\right)}<\frac{1}{s}$ by (12). By using a similar procedure in Theorem 2 , we can conclude that $f$ has a fixed point $z \in X$. Now, let $f u=u$. Then, from (11), we have

$$
q(u, u)=q(f u, f u) \preceq\left(\alpha_{1}+\alpha_{2}+\alpha_{3}+\alpha_{4}\right) q(u, u)
$$

which means that $q(u, u)=\theta$ by $(12)$ and $(*)$. This completes the proof.

Remark 6. Note that we also can obtain the proof of the Theorem 4 by considering $y=f x$ in (11) and applying Theorem 2. Moreover, assume that from conditions ( $i)$ and (ii) in Theorem 4 only $(i)$ is satisfied. By choosing proper $\alpha_{i}$, respectively, we can obtain Banach-type, Kannan-type, Cho-type [21,31,34] fixed point results with respect to $a w$-b-cone distance in tos-cone b-metric spaces as follows:

$$
\begin{aligned}
& q(f x, f y) \preceq \lambda q(x, y), \quad \lambda \in\left[0, \frac{1}{s}\right) ; \\
& q(f x, f y) \preceq \lambda(q(x, f x)+q(y, f y)), \quad \lambda \in\left[0, \frac{1}{s+1}\right) ; \\
& q(f x, f y) \preceq \alpha_{1} q(x, y)+\alpha_{2} q(x, f x)+\alpha_{3} q(y, f y), \quad s\left(\alpha_{1}+\alpha_{2}\right)+\alpha_{3}<1 .
\end{aligned}
$$

In Theorem 4 and Remark 6 , let $s=1$. We have the same results under a $w$-cone distance.

In the sequel, we discuss on the equivalence some $w$-b-cone distance and $w t$-distance introduced by Hussain et al. [9]. Let $V$ be an absolutely convex and absorbing subset of $E$. Remember the Minkowski functional $q_{V}(x)=\inf \{\lambda>0: x \in \lambda V\}$ for $x \in E$ is a semi-norm on $E$ [28]. Also, $W \subset V$ implies that $q_{V}(x) \leq q_{W}(x)$ for $x \in E$.

Now, if $(E, P)$ is an ordered tvs with solid cone $P$ and $e \in \operatorname{int} P$, then $[-e, e]=(P-e) \cap(e-P)$ will be an absolutely convex neighborhood of $\theta$ [29], and $q_{e}$ denotes its Minkowski functional $q_{[-e, e]}$. Also, int $[-e, e]=($ int $P-e) \cap(e-\operatorname{int} P)$. Moreover, $q_{e}$ is a non-decreasing function on $P$.

Theorem 5. Let $P$ be a solid cone, $e \in$ int $P$ and $q_{e}$ be the corresponding Minkowski functional of $[-e, e]$. Consider a tus-cone $b$-metric space $(X, d)$ with $a w$-b-cone distance $q$ on $X$, and a b-metric space $\left(X, d_{q}\right)$ with $d_{q}=q_{e} \circ d$. Then $p=q_{e} \circ q$ is a wt-distance on $X$.

Proof. We must prove the conditions of Definition 3 are hold. Since $q_{e}$ is a semi-norm and $q$ is a $w$-b-cone distance, we have

$$
q_{e}(q(x, z)) \leq s\left[q_{e}(q(x, y))+q_{e}(q(y, z))\right]
$$

for all $x, y, z \in X$, which implies that $p(x, z) \leq s[p(x, y)+p(y, z)]$. Thus, the condition $\left(w_{1}\right)$ of the Definition 3 holds. Now, let $x \in X$ and denote $f(y)=p(x, y)$. We show that $g$ is lower $b$-semicontinuous. Let $y, y_{n} \in X$ such that $y_{n}$ convergent to $y$ in $d_{q}$. It is equivalent to $y_{n} \rightarrow y$ in $d$. Since $q$ is lower $b$-semicontinuous, for given $\epsilon>0, q(x, y) \preceq s q\left(x, y_{n}\right)+\epsilon e$ for $n$ large enough. Since $q_{e}$ is a semi-norm, then $p(x, y) \leq s p\left(x, y_{n}\right)+\epsilon$; that is, $f=p(x, \cdot)$ is also lower $b$-semicontinuous. Thus, the condition $\left(w_{2}\right)$ of the Definition 3 holds. In order to proof the validity of $\left(w_{3}\right)$, select arbitrary $\epsilon$ and $\epsilon^{\prime}$, and set $c=\epsilon e$. Clearly, $c \in$ int $P$ and hence, by $\left(q_{3}\right)$, there exist $\theta \ll c^{\prime}=\epsilon^{\prime} e$ with $q(z, x) \ll c^{\prime}$ and $q(z, y) \ll c^{\prime}$ implying that $d(x, y) \ll c$. By Minkowski functional $q_{e}$, we have $p(z, x)<\epsilon^{\prime}$ and $p(z, y)<\epsilon^{\prime}$, which implies that $d_{q}(x, y)<\epsilon$. This completes the proof.

Note that all examples about the existence of fixed points in the framework of $w$-cone distance and generalized $c$-distance can be obtained in the version of $w$-b-cone distance by considering introduced distances in Example 2 and applying Theorem 5. 
A order $\subseteq$ is called a partial order or an order relation if it is reflexive, symmetric and transitive. Now, we consider the main theorem of Hussain et al. [9] in the framework of $w$-b-cone distance in ordered tvs cone $b$-metric spaces by applying the assertion of Theorem 5 .

Theorem 6. Consider a complete partially ordered tos-cone b-metric space $(X, d)$ with parameter $s \geq 1$ and $w$-b-cone distance $q$ on $X$. Assume that $f: X \rightarrow X$ is a non-decreasing mapping such that

$$
q\left(f x, f^{2} x\right) \preceq r q(x, f x)
$$

for all $x \subseteq f x$, where $r \in[0,1 / s)$. Also, suppose that the following conditions hold:

(i) for all $x \in X$ with $x \subseteq f x$, there exists $c \in$ int $P$ such that

$$
c \ll q(x, y)+q(x, f x)
$$

for each $y$ with $y \neq f y$;

(ii) there is $x_{0} \in X$ such that $x_{0} \subseteq f x_{0}$.

Then $f$ has a fixed point in $X$.

Proof. Let $e \in \operatorname{int} P$ and $q_{e}$ be a Minkowski functional, $p=q_{e} \circ q$ be defined by Theorem 5 and $d_{q}=q_{e} \circ d$ be defined by Lemma 4 of Kadelburg et al. [19]. Then, by $q_{e}$ and from (13), we have

$$
p\left(f x, f^{2} x\right) \preceq r p(x, f x)
$$

for all $x \in X$, where $r \in[0,1 / s)$. Also, by $q_{e}$ and (14), we obtain $\inf \{p(x, y)+p(x, f x)\}>0$ for all $x \in X$ with $x \subseteq f x$ and all $y$ with $y \neq f y$. Thus, the conclusion follows from Theorem 4.2 of [9].

Remark 7. Similar to the above theorem, one can obtain all results of Hussain et al.'s paper [9] in the framework of $w$-b-cone distance in ordered tos cone b-metric spaces by applying the assertion of Theorem 5. Also, in Theorem 6, set $s=1$. Then we can obtain this theorem in the framework of a w-cone distance.

\section{Conclusions}

In this paper, we defined the concept of a $w$-b-cone distance in tvs-cone $b$-metric spaces and discussed on its properties. Note that a $w$-b-cone distance with $s=1$ is a $w$-cone distance. Hence, the class of $w$-b-cone distances in tvs-cone $b$-metric spaces is bigger than the class of $w$-cone distances in tvs-cone metric spaces. Also, a generalized $c$-distance is a $w$-b-cone distance, but the converse is not true. Hence, the class of $w$-b-cone distances in tvs-cone $b$-metric spaces is bigger than the class of generalized $c$-distances in cone $b$-metric spaces. Our results are significant, since all of the results in fixed point theory with respect to a generalized $c$-distance can be introduced in the version of $w$-b-cone distance. Also, by Theorem 5 , if a theorem proved in the version of $w t$-distance, then this theorem is held in the version of $w$-b-cone distance. Thus, henceforth, the published results about fixed point theorems with respect to a $w$-b-cone distance or a generalized $c$-distance (a $w$-cone distance or a $c$-distance) in tvs-cone $b$-metric spaces (tvs-cone metric spaces) are not new and real unless they did not proved in the version of a $w t$-distance (a $w$-distance) in $b$-metric spaces (metric spaces). As a new subject, one can check this idea about distances in various cone $b$-metric spaces such as: generalized cone metric spaces, generalized cone $b$-metric spaces and etc.

Author Contributions: All authors contributed equally and significantly in writing this paper. All authors have read and agreed to the published version of the manuscript.

Funding: The authors are very grateful to the Basque Government by its support through Grant IT1207-19.

Acknowledgments: The first and the second authors acknowledges the Central Tehran Branch of Islamic Azad University. Also, the authors are very grateful to the Basque Government by its support through Grant IT1207-19 
Conflicts of Interest: The authors declare no conflicts of interest.

\section{References}

1. Wilson, W.A. On semi-metric spaces. Am. J. Math. 1931, 53, 361-373. [CrossRef]

2. Bakhtin, I.A. The contraction mapping principle in quasimetric spaces. Func. Anal. Gos. Ped. Inst. Ulianowsk 1989, 30, 26-37.

3. Boriceanu, M. Fixed point theory for multivalued contractions on a set with two $b$-metrics. Creative Math 2008, 17, 326-332.

4. Czerwik, S. Contraction mappings in b-metric spaces. Acta Math. Inform. Univ. Ostrav. 1993, 1, 5-11.

5. Khamsi, M.A.; Hussain, N. KKM mappings in metric type spaces. Nonlinear Anal. 2010, 73, 3123-3129. [CrossRef]

6. Kada, O.; Suzuki, T.; Takahashi, W. Nonconvex minimization theorems and fixed point theorems in complete metric spaces. Math. Japon. 1996, 44, 381-391.

7. Ilić, D.; Rakočević, V. Common fixed points for maps on metric space with w-distance. Appl. Math. Comput. 2008, 199, 599-610. [CrossRef]

8. Shioji, N.; Suzuki, T.; Takahashi, W. Contractive mappings, Kannan mappings and metric completeness. Bull. Am. Math. Soc. 1998, 10, 3117-3124.

9. Hussain, N.; Saadati, R.; Agarwal, R.P. On the topology and wt-distance on metric type spaces. Fixed Point Theory Appl. 2014, 2014, 88. [CrossRef]

10. Deimling, K. Nonlinear Functional Analysis; Springer: Berlin/Heidelberg, Germany, 1985.

11. Vandergraft, J.S. Newton's method for convex operators in partially ordered spaces. SIAM J. Num. Anal. 1967, 4, 406-432. [CrossRef]

12. Zabrejko, P.P. K-metric and K-normed linear spaces:survey. Collect. Math. 1997, 1997, 825-859.

13. Huang, L.G.; Zhang, X. Cone metric spaces and fixed point theorems of contractive mappings. J. Math. Anal. Appl. 2007, 332, 1467-1475. [CrossRef]

14. Du, W.S. A note on cone metric fixed point theory and its equivalence. Nonlinear Anal. 2010, 72, $2259-2261$. [CrossRef]

15. Janković, S.; Kadelburg, Z.; Radenović, S. On cone metric spaces; a survey. Nonlinear Anal. 2011, 74, 2591-2601. [CrossRef]

16. Azam, A.; Mehmood, N. Multivalued fixed point theorems in tvs-cone metric spaces. Fixed Point Theory Appl. 2013, 2013, 184. [CrossRef]

17. Hussain, N.; Shah, M.H. KKM mapping in cone b-metric spaces. Comput. Math. Appl. 2011, 62, 1677-1684. [CrossRef]

18. Cvetković, A.S.; Stanić, M.P.; Dimitrijević, S.; Simić, S. Common fixed point theorems for four mappings on cone metric type space. Fixed Point Theory Appl. 2011, 2011, 589725. [CrossRef]

19. Kadelburg, Z.; Paunović, Lj.; Radenović, S.; Soleimani Rad, G. Non-normal cone metric and cone b-metric spaces and fixed point results. Ser. A Appl. Math. Intorm. Mech. 2016, 8, 177-186. [CrossRef]

20. Du, W.S.; Karapinar, E. A note on $b$-cone metric and its related results: Generalizations or equivalence? Fixed Point Theory Appl. 2013, 2013, 210. [CrossRef]

21. Cho, Y.J.; Saadati, R.; Wang, S.H. Common fixed point theorems on generalized distance in ordered cone metric spaces. Comput. Math. Appl. 2011, 61, 1254-1260. [CrossRef]

22. Wang, S.; Guo, B. Distance in cone metric spaces and common fixed point theorems. Appl. Math. Lett. 2011, 24, 1735-1739. [CrossRef]

23. Đorđević, M.; Đorić, D.; Kadelburg, Z.; Radenović, S.; Spasić, D. Fixed point results under c-distance in tvs-cone metric spaces. Fixed Point Theory Appl. 2011, 2011, 29. [CrossRef]

24. Rahimi, H.; Soleimani Rad, G. Common fixed-point theorems and c-distance in ordered cone metric spaces. Ukrain. Math. J. 2014, 65, 1845-1861. [CrossRef]

25. Bao, B.; Xu, S.; Shi, L.; Ćojbasic Rajić, V. Fixed point theorems on generalized c-distance in ordered cone b-metric spaces. Int. J. Nonlinear Anal. Appl. 2015, 6, 9-22.

26. Fallahi, K.; Soleimani Rad, G.; Radenović, S. Algebraic distances in various algebraic cone metric spaces. Publications de l'Institut Mathématique 2018, 104, 89-99. [CrossRef] 
27. Soleimani Rad, G.; Rahimi, H.; Vetro, C. Fixed point results under generalized c-distance with application to nonlinear fourth-order differential equation. Fixed Point Theory 2019, 20, 635-648.

28. Schaefer, H.H. Topological Vector Spaces; Springer: New York, NY, USA, 1971.

29. Yau-Chuen, W.; Kung-Fu, N. Partially Ordered Topological Vector Spaces; Clarendon Press: Oxford, UK, 1973.

30. Kadelburg, Z.; Radenović, S.; Rakočević, V. A note on the equivalence of some metric and cone metric fixed point results. Appl. Math. Lett. 2011, 24, 370-374. [CrossRef]

31. Ćirić, Lj.; Lakzian, H.; Rakocević, V. Fixed point theorems for $w$-cone distance contraction mappings in tvs-cone metric spaces. Fixed Point Theory Appl. 2012, 2012, 3. [CrossRef]

32. Kadelburg, Z.; Radenović, S. Coupled fixed point results under tvs-cone metric and $w$-cone-distance. Adv. Fixed Point Theory 2012, 2, 29-46.

33. Rahimi, H.; Rhoades, B.E.; Radenović, S.; Soleimani Rad, G. Fixed and periodic point theorems for T-contractions on cone metric spaces. Filomat 2013, 27, 881-888. [CrossRef]

34. Soleimani Rad, G.; Fallahi, K.; Kadelburg, Z. New fixed point results under generalized c-distance in tvs-cone $b$-metric spaces with an application to systems of Fredholm integral equations. J. Math. Ext. 2018, 12, 1-19.

35. Abbas, M.; Rhoades, B.E. Fixed and periodic point results in cone metric spaces. Appl. Math. Lett. 2009, 22, 511-515. [CrossRef]

36. Jeong, G.S.; Rhoades, B.E. Maps for which $F(T)=F\left(T^{n}\right)$. Fixed Point Theory Appl. 2005, 6, 87-131.

37. Jeong, G.S.; Rhoades, B.E. More maps for which $F(T)=F\left(T^{n}\right)$. Demonstratio Math. 2007, 40, 671-680. [CrossRef]

(C) 2020 by the authors. Licensee MDPI, Basel, Switzerland. This article is an open access article distributed under the terms and conditions of the Creative Commons Attribution (CC BY) license (http://creativecommons.org/licenses/by/4.0/). 